\title{
Development, cell line differentiation and virulence in the primitive stem-/progenitor cell lineage of Entamoeba
}

\author{
Vladimir F. Niculescu* \\ D-86420 Diedorf, Kirschenweg, Germany
}

\begin{abstract}
Oxygen is the key regulator of cell differentiation and this applies not only to multi-celled organisms - from spongia to mammalian- but also to the primitive stem/ progenitor cell lineage of parasitic Entamoeba species. Strong hypoxia leads cells of the primitive stem/progenitor cell lineage of Entamoeba into a reversible state of non-proliferation. There are some reasons to assume that Entamoeba's cell lineage consist of more cell lines capable of asymmetric cell division than initially thought. As observed in OCB cultures the primary self renewing stem cell line of E. invadens (p-SRL) generates early and almost simultaneously two specialized progenitor cell lines: the secondary more oxygenic cell line s-SRL producing precursor cells for terminal differentiation (encystment) and the tertiary more hypoxic t-SRL cell line that give rise to quiescent cells for further differentiation. p-SRL and s-SRL are early non invasive (commensal) cell lines. Progressive oxygen depletion converses the early s-SRL into a trans-differentiated late td-SRL cell line. Persistent oxygen supplies prolong the life span of the s-SRL preventing transdifferentiation. The permanent t-SRL and td-SRL cell lines and their mitotic arrested cells are morphologically indistinguishable, however, they have different origin and pathogenic potential by different molecular organization, gene expression and transcriptional activity. We suppose that transdifferentiation activates epigenetic silent genes and see analogies between the t-SRL and td-SRL lines of E. invadens and the syngenic A and B cell lines of E. bistolytica.
\end{abstract}

\begin{abstract}
Abbreviations: OCB: oxygen consuming bacteria; SRL: selfrenewing cell line; ATD: autonomous terminal differentiation; ITD: induced terminal differentiation; p-SRL: primary self-renewing stem cell line; s-SRL: secondary self-renewing cell line; t-SRL: tertiary selfrenewing cell line; td-SRL: transdifferentiated cell line; RSC: reserve stem cells; MAS: mitotic arrested secondary cell; MAT: mitotic arrested tertiary cell; MA/td: mitotic arrested cells of the td-SRL; A: amoebulae, M: metacyst, ILH: identical low hypoxic daughter cells ; ISH: identical strong hypoxic cells
\end{abstract}

\section{Stem cell lineage and life cycle of Entamoeba}

Recently, a primitive stem cell protolineage comprising an undifferentiated self renewing stem cell line and more specialized progenitor cell lines was discovered in OCB cultures of Entamoeba invadens [1-3]. In vitro OCB cultures reproduced the whole multilined life cycle of Entamoeba as it occur in the host intestine. Each self renewing cell line (SRL) contains a constant, invariable quantity of cells from which asymmetric cell divisions are always able to assure self-renewal. A separate work will demonstrate how OCB cultures lead Entamoebae into asymmetric cell division.

Most likely, similar protolineages exist in other intestinal pathogen protists such as E. histolytica, E. dispar and Giardia [4,5] and lead to the assumption that the basic molecular mechanisms of stemness conserved in anaerobe protists are inherited from the eukaryotic last common ancestor. Stemness features inherited by modern day protists facilitate their evolution into the parasitic way of life [6]. The dogma considering that stem cells appear first in multi-celled organisms such as Porifera (Spongia) and Cnidaria (Hydra) [7] is no longer supported by these observations.

The primitive cell lineage of Entamoeba contains multiple vegetative cell types and cell lines of various differentiating potential capable of encystment or not. It completes the insufficient life-cycle concept of Entamoeba. There is no exact match between the primitive protolineage of Entamoeba and the highly evolved stem cell lineages of mammalians and humans. However, both stem cell systems possess the main properties defining stem cells: (1) stem cells proliferate indefinitely by self-renewal maintaining the unspecialized (undifferentiated) cell state, (2) reserve stem cells replenish the primitive stem cell line (3) stem cells can change into specialized cells and subsidiary cell lines with a more limited potential (potency restriction) (4) all cell lines give rise to mitotic quiescent cells capable of further differentiation.

In the human stem cell terminology the terms "stem cell" and "progenitor cell" are sometimes equated, however, progenitor cells are already more specific than stem cells and differentiate into "target" cells. Progenitor cells are in an intermediary position between stem cells and fully differentiated cells. The majority of progenitor cells lie dormant (mitotic quiescent) or possess little activity in the tissue in which they reside. They grow by slow proliferation and start differentiation into target cells.

In the highly evolved stem cell systems of mammalians, progenitor cells can divide only a limited number of times. In contrast, progenitor cell lines of Entamoeba are highly controlled by extrinsic signals such as oxygen. Some of these cell lines (s-SRL) have an oxygen dependant life span while other cell lines (t-SRL) implicated in invasiveness and pathogenicity can survive indefinitely (permanent cell lines).

Correspondence to: Niculescu VF, Kirschenweg 1, D-86420 Diedor (Bayern) Germany, Tel: 0049-8238-4346; E-mail: vladimir.niculescu@yahoo.com

Key words: Entamoeba invadens, E. histolytica, transdifferentiation, pathogenicity, oxygen

Received: January 22, 2017; Accepted: February 06, 2017; Published: February 09,2017 


\section{Intestinal oxygen gradients, OCB cultures and lineage development}

Living in the intestinal tract of their host, amoebae such as $E$. histolytica, E. dispar and E. invadens benefit from the oxygen gradients occurring between the anoxic lumen and the pericapillar zones of invaded tissues and organs [4]. In the host's intestine a multitude of oxygen zones ranging from $\leq 0.1 \%$ to $5.5 \%$ O2 control amoebic development. These oxygen values correspond to the hypoxic and less hypoxic niches that control stem cell development in mammalians and humans.

Oxygen consuming bacterial sediments (OCB sediments) mimicking intestinal microconditions were developed to induce asymmetric cell fate as an alternative to axenic cultures [2,3]. We used metabolically repressed bacterial sediments of Aerobacter aerogenes containing 5-15 mg bacteria to mimic all zones of the intestinal oxygen gradient (oxygenic, low/mid hypoxic, strong hypoxic). OCB sediments reduce the oxygen content to an appropriate hypoxic content $(<5.5 \%$ ) capable to start amoebulae into the asymmetric cell fate and multiple differentiation processes not occurring in axenic cultures (oxygen content $>5.5 \%$ ).

Under low/mid hypoxic conditions ( $3-5.5 \%$ O2 content) all cell lines of E. invadens proliferate by asymmetric division keeping the number of self-renewing cells constant. Population increase takes place only by accumulation of uncommitted mitotic arrested cells (MAP and MAT cells) respectively committed precursor cells for autonomous terminal differentiation (MAS cells). Oxygen depletion prolonged the duration of the cell cycle from 5-6 hrs (fast cycling) in the upper oxygen gradient zones, to about $24 \mathrm{hrs}$ (slow cycling) in low/mid hypoxic zones. Strong hypoxic zones and anoxia stress amoebae. They switch consequently from asymmetric to symmetric cell division and arrest in a G2/G0 state waiting for re-oxygenation [4].

In the past the greatest impediment to understanding Entamoeba's stemness potential was the axenic culture method. Axenic cultures are best suited for biochemical research but less suitable for cell biological studies. In the absence of OCB associates amoebae grow in oxygen contents $>5.5 \%$. Axenic cultures repress asymmetric cell fate and do not allow differentiation events. Daughter cells are apparently identical and ATD cysts were no formed. Consequently, the primary stem cell line p-SRL and the late td-SRL cell line are missing in axenic cultures. The major and minor subpopulations observed in axenic cultures of $E$. histolytica correspond to symmetric proliferating s-SRL and t-SRL cell lines [8]. After symmetric cell division the daughter cells of the minor subpopulatiion become polyploid. Defective centrosome division leads to multipolar spindle formation, defective mitosis and a state of reversible non-developmental polyploidy. This reversible polyploidy is caused by defective mitosis $[4,8,9]$ and not by the lack of checkpoints as incorrectly proposed in the past [10].

\section{Excystment and early cell lines}

The life cycle of E. invadens begins by excystment and dedifferentiation of the dormant tetranucleated innercyst cell (ICC) containing the eight genome copies (Figure S1, supplement). In the early developmental phase the hatched ICC cell (metacyst, M cell) segregates its genome copies into eight daughter cells (amoebulae /A cells) by reductive nuclear divisions, nuclear seggregation and cellularization $[8,9]$. The mononucleated $1 \mathrm{C}$ amoebulae are totipotent cells that form the primary self renewing cell line p-SRL.
The p-SRL is a pluripotent like cell line consisting of undifferentiated cells. It proliferates and divides in OCB cultures asymmetrically producing self-renewing and mitotic arrested MAP cells as reserve stem cells (RSC). RSCs replenish in subculture the p-SRL line. The natural habitat of the p-SRL is the small intestine [4]. It cannot be excluded that $\mathrm{p}$-SRL find in vivo conditions to proliferate by symmetric cell divisions amplifying the bulk of undifferentiated primitive stem cells by a multiple of 8 . That could be a possible explanation why only a few cysts may successfully initiate major infections.

The primary $\mathrm{p}$-SRL converts by extrinsic mechanisms of signaling and transduction to two alternate and disparate identities forming specialized progenitor cell lines. One is the secondary unipotent s-SRL producing committed precursor cells for autonomous terminal differentiation (Figure S1). It is an oxygenic cell line that proliferates in the upper zones of the intestinal oxygen gradients. The other is the tertiary t-SRL lacking the capacity to produce cysts in cultures. In contrast, the vegetative $\mathrm{t}$-SRL produces mitoticly arrested tertiary cells (MAT cells) capable of further maturation and differentiation [1-3].

\section{Long living cell lines}

The early p-SRL and s-SRL cell lines are short leaving cell lines. Oxygen variation converts these cell lines in two permanent lines capable of unlimited proliferation in all gradient zones. One is the permanent t-SRL originating from the pluripotent $\mathrm{p}$-SRL and the other is the vegetative cell line td-SRL originating from the unipotent s-SRL. As previously described low/mid hypoxic conditions switch the s-SRL into a late vegetative cell line (td-SRL) morphologically indistinguishable from the t-SRL $[1,3,4]$. Both permanent cell lines produce mitotic quiescent cells but no cysts. However, they have different origin: the early $t$-SRL originates from the pluripotent $\mathrm{p}$-SRL stem cell line while the late $t d-S R L$ arises from the s-SRL by cross differentiation (transdifferentiation).

t-SRL and td-SRL have not only different origins but also different "birth dates" and different pathogenic potential. While the t-SRL is an early cell line concomitant with the s-SRL or preceding it, the tdSRL is an obligate post-encystment cell line. It comes in existence as encystment breaks down and the cyst producing s-SRL line is released (Figure S1). We consider the p-SRL and s-SRL lines as pre-invasive commensal cell lines (short living cell lines), while both the t-SRL and td-SRL are potentially invasive lines capable of amoeboid migration in the host tissues and organs. Especially mature MAT and MA/td cells are suspected to be highly hematophagous and invasive.

\section{Oxygen is the key regulator of development and proliferation}

Amoebic development and differentiation follow genetically determined programs encoded in the amoebic genome and controlled by extrinsic cues particularly by oxygen. It was reported that cytopathic activity increases in high oxygenic environments [11]. On the other side hypoxia activates several other molecular pathways and transcriptional programs [12].

Lineage development starts in the primary OCB culture by excystment, reductive metacystic division and de-differentiation induced by stimuli that mimic environmental conditions from the small intestine. The final products of ex-cystment are the eight juvenile amoebulae (Figure S1). This juvenile cell fraction maintains the totipotency of the mature innercyst cell (ICC) and hatching metacysts (M cell). 
Genome activation occurring during ex-cystment is similar to the genome activation occurring during zygotic cleavage [13]. As known, the totipotent mammalian zygote divides and creates totipotent replicas. Totipotency persists in mammalian embryos also until the eight cell zygote stage. From that stage on, genome undergoes activation (maturation) and differentiation forming pluripotent and multipotent cell types [14-16]. Eukaryotic development is a story of self-renewing events, potency restriction and cell fate specification by mechanisms inherited from the common ancestor. Development continues by morula, blastocyst and the innercyst cell mass/epiblast (ICM) that gives rise in humans and mammalians to embryonic pluripotent stem cells that grow indefinitely maintaining pluripotency and differentiation potential.

\section{From amobulae to primitive stem cells living in low oxygen environments (hypoxic a/p conversion)}

Amoebulae (A cells) are a highly regulated cell stage that activates stemness, lineage development and totipotency restriction. During $\mathrm{a} / \mathrm{p}$ conversion amoebulae converted into the primitive pluripotent stem cell line p-SRL that is the portal for subsequent development.

Developmental lineages are controlled by evolutionarily conserved transcription factors and transcriptional regulatory circuits [17]. In multi-celled organisms, transcription factors such as OCT4 and NANOG are essential regulators of early development, stem cell identity and self-renewal; they play a key role in cell fate specification [17-18]. A multitude of small proteins regulate signalling and epigenetic mechanisms in differentiation. Cell identity is controlled by target genes for transcriptional regulators and auto-regulatory gene expression programs.

The primitive stem cell line p-SRL starting in low/mid hypoxic OCB sediments is very sensitive to variations of the surrounding oxygen content. It perceives changes of oxygen pressure (pO2) corresponding to the gradient zones below or above its own oxygen zone and responds to these changes by appropriate cell conversion. $\mathrm{pO}_{2}$ changes activate specific transcription factors steering subsequent lineage development. In OCB cultures the p-SRL is a short living stem cell line. Oxygen changes occurring by aeration or sub-culturing switch the p-SRL either into the secondary s-SRL or tertiary t-SRL, connecting signalling pathways on pluripotency reduction and further differentiation.

\section{From primitive stem cells to the early secondary cell line $s$-SRL (oxygenic p/s conversion)}

$\mathrm{p} / \mathrm{s}$ conversion occurs either by aeration of the primary OCB culture or when primary cells are transferred in subcultures with < $5 \mathrm{mg}$ A. aerogenes [3]. Culture passaging is a short exposure to oxygen contents $>5.5 \%$. $\mathrm{p} / \mathrm{s}$ conversion turns off genes of the pluripotent cell state and restricts genome expression to an unipotent differentiation program expressed by committed MAS cells that are precursors of ATD encystment.

s-SRL is an oxygenic cell line. It proliferates in young OCB cultures as long as oxygen depletion did not exceed a critical value of low hypoxia [2,3]. p/s conversion activates fast cycling (cell cycle duration: 5-6hrs) and autonomous terminal differentiation (ATD). The molecular mechanisms of this oxygenic program were developed by the anaerobe common ancestor LECA at the time oxygen increased to about $5 \% \mathrm{O}_{2}$. Thus, the s-SRL corresponds to an evolutionary "young" cell line specialized for reproductive encystment and cysts dissemination - despite abundant nutrient supply. Evolutionarily, it is the transition from extrinsic to intrinsic differentiation. In return, oxygen depletion stops the process of autonomous encystment: this includes polyploidisation, maturation and progression through the TD checkpoint [9]. To continue differentiation arrested MAT precursors need re-oxygenation.

\section{From primitive stem cells to the early tertiary cell line $t$-SRL (hypoxic p/t conversion)}

In contrast, the $\mathrm{p} / \mathrm{t}$ conversion takes place as the $\mathrm{p}$-SRL line was transferred into more hypoxic culture sediments containing $>5 \mathrm{mg}$ A.aerogenes. In other words, the early t-SRL comes about in conditions of low/mid hypoxia. Reduced oxygen supply is a relevant factor not only in E.invadens' development but also in hematopoiesis and malignant diseases [19].

Subpopulations produced by the ubiquitous proliferating t-SRL line reside in all zones of the intestinal oxygen gradient mimicked by OCB sediments. They conserves stem cell features and molecular mechanisms developed by LECA in a previous evolutionary period of less oxygen content and is much more subject to extrinsic stimuli. Accordingly, strong hypoxic environments and anoxia $\left(<1 \% \mathrm{O}_{2}\right)$ lead the t-SRL line to symmetric cell division, identical daughter cell formation, cell line amplification and finally to proliferation arrest (ISH cells). Mid hypoxic ranges $\left(2-3 \% \mathrm{O}_{2}\right)$ led to slow cycling while slow hypoxic and upper oxygenic values $\left(4-5.5 \% \mathrm{O}_{2}\right)$ lead the $\mathrm{t}-\mathrm{SRL}$ line to fast cycling. Fast and slow cycling are regulated by oxygen increase and oxygen depletion [3]. Extrinsic signalling controls self renewal.

In conditions of strong hypoxia signalling controls self-renewal. In conditions of strong hypoxia (SH) the t-SRL switch to complete self renewal (symmetric division) forming ISH cells that arrest in a G2/M state as long hypoxia persists. When hypoxia declines the G2/M cells continue asymmetric proliferation first by slow cycling (cell cycle duration: $\geq 24 \mathrm{hrs}$ ). Further oxygenation leads the t-SRL lines back to fast cycling (cell cycle duration: $\sim 6 \mathrm{hrs}$ ); in upper oxygenic zones the t-SRL cells cycled nearly as fast as the s-SRL. Fast cycling by oxygen content increase is an evolutionary improvement of proliferation in anaerobe Entamoebae [3,9].

Lacking mechanisms of further conversion, the early t-SRL line may proliferate ad infinitum. It lacks the capacity to convert back to a pluripotent stem cell state. Mitoticly arrested MAT cells are not committed for encystment however, they can be manipulated to form cysts $[3,8,9]$. Unfavourable life conditions such as nutrient deficiency and hypo-osmotic media induce G1 and G1/G0 cells directly to a self guarding terminal differentiation (ITD), while G2/M cells finish the current cell cycle by symmetric division producing two identical low hypoxic daughter cells (ILH cells) capable of ITD encystment. Encystment and totipotency recovery occurs in starving high density OCB sediments ( $15 \mathrm{mg} \mathrm{A}$. aerogenes as $3 \mathrm{xOCB}$ dose) that mimic oxygenic zones favourable to encystment. Extrinsic signalling and nutrient deficiency reactivates the ancestral mechanisms of extrinsic controlled ITD encystment. Thus, it is clear that the t-SRL line - not producing ATD cysts in cultures - preserves a latent primitive gene program that may be activated by extrinsic stressors. Starving cells prior the RP checkpoint (ILH and G1 cells) commit to precursor cells for terminal differentiation.

In vivo, the t-SRL line may sometimes precede the phase of cyst formation and dissemination. We believe that $\mathrm{p} / \mathrm{t}$ conversion take place when the primary stem cell line enters mid oxygenic zones at 
the surface of the colon mucous layer. Initial stages of amoebiasis and "healthy" carriers of E. histolytica cysts lead to the assumption that the early t-SRL line is initially not particularly invasive.

\section{From the early s-SRL to the late cell line td-SRL (hypoxic transdifferentiation)}

This cell line conversion is the unique differentiation form not occurring from the pluripotent stem cell state (p-SRL cells). It is rather a form of primitive transdifferentiation starting from a fully committed cell line, namely the unipotent s-SRL. We name this late transdifferentiated cell line (originating from the s-SRL) the td-SRL line. Similarly with the t-SRL, the td-SRL that gives rise to not committed mitotic quiescent cells in a G0/G1 cell state (MA/td cells) that may reenter the mitotic cell cycle for further proliferation. Morphologically the td-SRL and the t-SRL are indistinguishable. Both cell lines proliferate indefinitely producing apparently identical quiescent cells (G0/G1cells). However, the td-SRL and t-SRL cell lines have different origins, "birth dates" and a cell fate that led to the assumption that the td-SRL is strongly implicated in the invasive amoebic disease (virulent phenotype).

Transdifferentiation occurs in vitro as the oxygen content decreases to hypoxic ranges improper for s-SRL proliferation and cyst formation, in other words as ATD encystment breaks down. s-SRL cells convert to the td-SRL cell line, breaking the cell line commitment fidelity. Transdifferentiation is genome re-programming without going through the pluripotent cell state p-SRL. Concluding, the unipotent $\mathrm{s}$-SRL line preserves cell plasticity and a latent invasive gene expression program that can be activated by oxygen depletion.

It is well known that low oxygenic stimuli and signaling modulator pathways promote direct reprogramming in stem cell systems [20]. In Entamoeba the unipotent cells of the s-SRL line have the opportunity to increase their developmental potential directly, without going back to a state of less differentiated pluripotency. Reactivating silenced genes, the unipotent s-SRL line switches directly into another cell type. It is a case of natural primitive transdifferentiation occurring by extrinsic signaling. The loss of lineage commitment [21] allows s-SRL cells to switch into another phenotype.

In the recent years, the transdifferentiation of stem and progenitor cells was considered controversial and its biological significance is still poorly understood [22]. Transdifferentiation means irreversible conversion of cells from one cell type to another [23]. Natural transdifferentiation is rare in highly evolved mammalian cells, it does occur only in situations where missing limb or organs regenerate in organisms. Most frequently, transdifferentiation occurs by experimental manipulation or de-differentiation. During reprogramming the epigenetic specification will be erased or remodeled [24-25]. Good evidence for natural reprogramming and transdifferentiation were found in the zygote to blastocyst development, as cells have to make crucial decisions important for later development $[23,26]$.

\section{Checkpoints of cell cycle progression and development}

As mentioned above t-SRL cells passaging the restriction point RP are committed for mitotic progression and can not be induced to form ITD cysts $[3,8,9]$.

\section{Cell cycle surveillance}

Cell cycle progression is controlled by surveillance mechanisms specific for eukaryotic cells. These surveillance mechanisms consists of check points ensuring that cycling cells will not progress the next phase of the cell cycle or development before the preceding phase have been completed [27-30]. G1, S and G2 checkpoints arrest cell cycle progression in response to detecting an inadequate supply of nucleotides or other resources necessary for mitotic progression. They also ensure that the replication process is not derailed by damaged $\mathrm{DNA}^{*}$. Similarly, checkpoints prevent cycling cells from returning back to a previous cell cycle phase. Progress in checkpoints and surveillance research has come in recent years from cancer cell research.

Checkpoints are regulatory molecular steps that decide if a cell will enter and progress mitotic cell cycle or not. Accordingly, interdependent regulatory transitions bring Entamoeba's DNA in a state competent (i) for cell cycle progression and cell division or (ii) for endoreplication and terminal differentiation. Commitment to another round of replication depends of a number of extrinsic and intrinsic conditions such as the presence of sufficient nutrients and synthesis of proteins and nucleotide precursors necessary for genome duplication*. Whether a cell commits to mitotic progression and cell division depends ultimately on RP decisions.

${ }^{*} J o n a t h a n$ M (2013) The Cell's Surveillance System: Introducing the Cell Cycle Checkpoint Pathways. http://www.evolutionnews. org/2013/08/the_cells_surve075151.html

Especially in the case of t-SRL cells it becomes evident how the network of interacting checkpoint pathways operates in concert to lead mitoticly committed G1 cells to mitosis and cell division: cells passaging the RP checkpoint are not capable to differentiate terminally to ITD cysts. Another eloquent result of Entamoeba research is the finding that ILH daughter cells - born in nutrient free media - as well as early G1 and quiescent G1(G0) cells transferred in nutrient free media contain sufficient amounts of maternal nucleotide precursors needed for endopolyploidisation, genome copying (8C) and encystment.

An important check point outside mitotic cell cycle is the TD check point that controls polyploid cell entry to terminal differentiation [9]. Low oxygen environ ments slow down polyploidisation and subsequent maturation. Immature polyploids arrest at the activated TD checkpoint as long as the requirements for terminal differentiation are not fullfilled.

\section{G1 decisions for cell line conversion and commitment}

Cell line conversion turns off genes of the current program needed for surviving in the new environmental conditions. Each cell conversion requires sets of transcription factors that exert a regulatory influence on the other differentially expressed genes in the local network [31]. Conversion selects an optimal set of transcription factors with the greatest influence over genes differentially expressed in the converted cell type in contrast to the primitive undifferentiated cell state. With each conversion, genes belonging to the current developmental program are repressed while genes responsible for the subsequent developmental program are activated. In Entamoeba the primitive pluripotent stem cell line p-SRL turns on a restricted number of genes; other gene families of the developmental programs are kept inactive.

The early G1 phase plays a crucial role in stem cell differentiation [32] and cell line conversion. Early G1 cells have mechanisms of signaling transduction and translate extrinsic signaling stimuli into specific developmental responses. In mammalians, undifferentiated pluripotent stem cells have a short G1 phase while committed cells extend it. Stem cells exposed to specific cell fate stimuli activate specific G1 developmental genes and programs that respond to prodifferentiation signals by cell cycle lengthening, changes in the 
epigenetic landscape [33] and asymmetric division.

In mammalians, asymmetric dividing stem cells need more time for cell cycle proliferation than symmetric proliferating cells. The lengthening of the cell cycle during stem cell differentiation is associated with distinct molecular kinetics [34]. Proliferating embryonic stem cells ESC of mammalian have a very short G1 phase (2 - 3hrs) [34] however, during stem cell differentiation the G1 phase is markedly elongated [35-36]. In neural stem/progenitor cells (NSC) the G1 phases doubles its length when induced to differentiation and the doubled G1 state promotes differentiation. However, it is not clear if G1 lengthening is the consequence or a cause of differentiation.

Cell cycle extension was not observed in committed E. invadens cell lines [3]. On the contrary, the oxygenic progenitor cell line s-SRL has the shortest cell cycle of the lineage (5-6 hrs). This cell line has, however, transdifferentiation capacity; it is not irreversible committed. Cell cycle prolongation was observed in the proliferation of the irreversible committed t-SRL line, however, this cell cycle extension is a response to oxygen depletion. G1 extension is probably an achievement of higher evolved stem cell systems specific for mammalians and humans.

\section{Genome reprogramming and epigenetic changes lead to virulence and invasiveness}

Differentiation is regulated by signalling proteins, epigenetic changes (histone hyperacetylation, DNA demethylation) and specific DNAses [37]. In differentiated cells, part of the chromatin is transcriptionally silent and other parts transcriptionally active. To understand the molecular mechanisms underlying specific gene regulation of different cell lines it is essential to understand the changes occurring in nuclear chromatin organization, chromatin folding and histone distribution [37].

We believe that Entamoeba's invasiveness and pathogenesis are related to epigenetical changes and cell line conversion occurring during late phase of development. Genome reprogramming by "epigenetic patterns" is a response to extrinsic signals. Intrinsic transduction mechanisms reprogram amoebic cells by "epigenetic patterns" [4].

Reik said in 2007 "development is, by definition epigenetic" [38]. This means, during early development stages genes required in later development are transiently held in a repressed state by certain histone modifications. Histone modifications play fundamental roles in gene silencing: they are highly flexible, reversing easily when silent gene activation is needed. The early development phase is a state of flexible epigenetic silencing [38], while late developmental stages differentiation narrows functional (invasive) potential.

We assume that intestinal t-SRL and td-SRL lines activates during the late developmental phase silencing invasiveness genes in an epigenetic fashion. These epigenetic changes occur in responses to luminal stimuli. We believe that extrinsic cues play a key role in activating silent genes correlated with high pathogenic and invasive activity [4].

\section{Intestinal and extra-intestinal genotypes}

More and more authors agree in recent years that the acquisition of invasiveness is accompanied by changes in gene expression and expression of specific genes [39], however, these changes could not be explained in detail. Origin, causes and interrelations between the observed genotypes could not be clarified. Numerous findings reviewed by Oliveira, et al. [40] described genetic diversity in E. histolytica and $E$. dispar relying on genetic differences observed in one and the same patient between stool and liver abscesses samples [40] or between distinct liver abscess foci [41]. These findings support the idea that different invasive outcomes originate from intestinal cell types of reduced pathogenic potential (diarrhoea and colitis) switch to the extraintestinal cell types producing amoebic liver abscesses (ALA) [42]. It was suggested that the outcome of infection may be determined by the parasite genotype. Phenotypes from the paired intestine/ ALA samples were considered genetically different. This leads to the question is there an intrinsic reorganization or recombination of amoebic DNA or an extrinsic induced genotyping*.

\section{*http://www.medscape.com/viewarticle/737810_6}

Already Ali et al $[43,44]$ revealed that intestinal and extraintestinal cell types are genetically distinct. The authors mean DNA reorganisation would take place "prior to or during metastasis from intestine to liver". The authors analysed intestinal and extra intestinal subpopulations by PCR and found a serine rich protein gene (SREHP) [44-46] that encodes an immuno-dominant surface antigen; intestinal amoebae and ALA amoebae were different from each other at this locus [44]. Authors supposed: (i) the original intestinal infection may have contained multiple genotypes of which only one migrates to the liver (as a bottleneck in parasite genotypes) or (ii) DNA reorganisation take place when amoebae migrate from the intestine to liver [44]. We believe that the existence of multiple intestinal genotypes is the result of a permanent regeneration of the amoebic lineage by reserve stem cells (RSC) of the niche, colonic re-excystment of intestinal just formed cysts and transdifferentiation.

Other authors favour the "genetic bottleneck" [47] or demographic sweep [48] hypothesis or suppose that certain microbiome features lead to differences in the outcome of E. histolytica infections $[49,50]$. Other authors conclude that "parasite genetics contribute significantly to virulence, and in particular, not all strains are capable of causing liver abscesses". On the other side two different ALA-genotpes were found in simultaneous but independent liver abscesses from the same patient [41].

Our findings in E. invadens confirm the last hypothesis. We consider the late td-SRL line and its mitotic arrested MA/td progeny represent the high pathogen phenotype and the early t-SRL/ MAT cell fraction - occurring concomitantly or preceding encystment - the less pathogenic phenotype. In E. histolytica cysts were found not only in asymptomatic individuals [51-53] but sometimes occur in ALA patients free of intestinal symptoms [54] where transdifferentiation could not be held: in such cases the s-SRL line prolonged its life span and only cells belonging to the t-SRL/MAT subpopulation could migrate to liver and destroy hepatic structures. However, it cannot be excluded that new amoebic lineages started by reserve stem cell or by amoebulae originated from re- excystment pathways (excystment of intra-colonic formed cysts) also cooperate for invasive amoebiasis.

\section{t-SRL and td-SRL cell lines and their syngenic relatives described in E. histolytica}

Despite their different origin the early t-SRL and the late td-SRL have many similarities to each other. Both are irreversible committed and indefinite proliferating cell lines (permanent cell lines) not leaving mitotic cell cycle. They proliferate in OCB cultures asymmetrically differentiating mitotic quiescent cells. In more hypoxic cultures sediments the early t-SRL may precede the s-SRL cell line, while the late td-SRL appears as encystment break down. 
In our opinion t-SRL and td-SRL of E. invadens are the relatives of the syngenic A and B cells recently described in E. histolytica [55-56]. The term syngenic (syngeneic) comes from the tumor and transplant medicine and means genetically identical respectively sufficient identical for transplantation (immunological compatible). When the cells are collected from the same patient they are called autologous, when collected from identical individuals they are referred as syngeneic.

Malignant and normal cell lines isolated from the same organ (providing several unique features) are also considered syngeneic although they are different in origin [57]. Syngeneic cell lines maintain a stable morphological and biological phenotype; the gene expression levels of syngeneic cell lines are stable over multiple generations [58].

Syngenic cell lines of E. histolytica derive from the pathogenic strain HM-1: IMSS strain. The A cell line is described as a nonpathogenic line, because incapable to produce lasting ALAs in gerbils, while cell line B leads to considerable abscesses [55]. According to the authors A cells have increased hemolytic activity, lower growth rate and smaller cell size, while $B$ cells are larger in size. Proteomic analyses show 21 proteins with higher abundance in the cell line $\mathrm{A}$ and 10 proteins with higher abundance in the cell line $\mathrm{B}$. There are significant and reproducible transcriptional differences between the two cell lines.

More recently the authors identified autologous A and B cell lines from a unique clinical isolate of the HM-1:IMSS strain and cloned them [56]. All A1-A12 clones are not capable of inducing ALAs in gerbil; therefore they are considered nonpathogenic. This "nonpathogenicity" includes however, the induction of small and quickly resolved hepatic lesions [56].

Interestingly, 4/12 of the pathogenic B clones have significantly reduced ability to induce ALAs in gerbils; one B clone (B8) did even not induce ALA formation and was also considered nonpathogenic. Conclusively, the authors above considered that the pathogenic HM1: IMSS strain has different molecular mechanisms leading to loss of pathogenicity. On the other side they report that the nonpathogenic A profile and the pathogenic B8 profile remain stable for years without animal passages; inter-conversion is not possible. Stable for years means both cell lines are irreversible committed permanent cell lines, not capable of transdifferentiation.

This finding suggests a clear analogy between the syngenic A and B cell lines of E. histolytica and the t-SRL and td-SRL lines of $E$. invadens. It is evidence that Entamoeba's permanent cell lines have mechanisms of virulence variation controlled by extrinsic stimuli and stressors. No information exists about whether the A cell lines are really nonpathogenic for human liver cells and unable to produce ALAs although recent findings suggest the participation of genetically distinct cell lines (genotypes) in individual ALA patients [41].

Similar to the t-SRL of E. invadens the A cell lines of E. histolytica are early developmental cell lines analogous to the early t-SRL while the late td-SRL is more analogous to the B line of E. histolytica. An open question is the origin of the autologous A and B cell lines (derived from a unique clinical isolate) [56]. Two alternative hypotheses would be conceivable: (i) either the isolate contains both A and B cell fractions when harvesting or (ii) it contains a mixture of transient p-SRL and s-SRL cells capable of further conversion to permanent A and B cell lines.

\section{Concluding remarks}

Oxygen is undoubtedly the key regulator of Entamoeba's stem cell lineage and life cycle development. It works as a microenvironment signal. In the past very little attention was given to the oxygen dissolved in culture media and its effect on the biology of Entamoebae. First OCB cultures with metabolically repressed $A$. aerogenes sediments lead to the discovery of the stem cell lineage and asymmetric cell fate in E. invadens and revealed the key role of intestinal oxygen gradients $\left(0.1 \%-5.5 \% \mathrm{O}_{2}\right.$ content $)$ on lineage dynamics. Self renewal, cell line differentiation and transdifferentiation are regulated both in vitro and in vivo by the surrounding oxygen amount. Extrinsic signaling mechanisms and intrinsic transduction mechanisms control Entamoeba's response. Upper oxygen gradient zones promote stem cell conversion into the unipotent progenitor cell line s-SRL that produces committed precursor cells for autonomous terminal differentiation (ATD encystment). Differentiation steps such as endoreplication, polyploid maturation and transiting through the checkpoint of terminal differentiation (TD check point) are oxygenic processes underlying oxygenic signals which do not exceed the 5.5\% O2 limit. While p-SRL and s-SRL are short living cell lines, the long living t-SRL and td-SRL are permanent and ubiquitous cell lines capable to survive and self-renew in all tissue and organs (Figure S1). Both cell lines have pathogenic potential, expressing different degrees of virulence. Variation in virulence expression is possible.

\section{Aknowledgements}

The author expresses his gratitude to Dr. Dennis Thomas (native English speaker) for reading of the manuscript and excellent support.

\section{References}

1. Niculescu VF (2014) The three line PST stem cell system of Entamoeba invadens as one of the earliest stem cell systems today.

2. Niculescu $\operatorname{VF}(2013)$ Growth of Entamoeba invadens in sediments with metabolically repressed bacteria leads to multicellularity and redefinition of the amoebic cell system. Roum Arch Microbiol Immunol 72: 25-48. [Crossref]

3. Niculescu VF (2015) The stem cell biology of the protist pathogen Entamoeba invadens in the context of eukaryotic stem cell evolution. Stem Cell Biol Res 2: 2.

4. Niculescu VF (2016) Pathogenicity of Entamoeba Species Depends on Cell Line Conversion, Genome Reprogramming and Epigenetic Gene Regulation. J Cell Sci Ther 7: 245

5. Niculescu VF (2014) The cell system of Giardia lamblia in the light of the protist stem cell biology. Stem Cell Biology and Research 1: 3 .

6. Niculescu VF (2014) The evolutionary history of eukaryotes: how the ancestral protolineage conserved in hypoxic eukaryotes led to protist pathogenicity. Microbiol Discov 2: 4.

7. Funayama N (2010) The stem cell system in demosponges: insights into the origin of somatic stem cells. Dev Growth Differ 52: 1-14. [Crossref]

8. Niculescu VF (2016) Developmental and Non Developmental Polyploidy in Xenic and Axenic Cultured Stem Cell Lines of Entamoeba invadens and E. histolytica. Insights Stem Cells 2: 1 .

9. Niculescu VF (2016) Low-oxygen environments slow down precursor cell developmen in the pathogen anaerobe protist Entamoeba invadens. J Stem Cell Res Med 1: 27-35.

10. Banerjee S, Das S, Lohia A (2002) Eukaryotic checkpoints are absent in the cell division cycle of Entamoeba histolytica. J Biosci 27: 567-572.

11. Akbar MA, Chatterjee NS, Sen P, Debnath A, Pal A, et al. (2004) Genes induced by a high-oxygen environment in Entamoeba histolytica. Mol Biochem Parasitol 133: 187196. [Crossref]

12. Mohyeldin A, Garzón-Muvdi T, Quiñones-Hinojosa A (2010) Oxygen in stem cell biology: a critical component of the stem cell niche. Cell Stem Cell 7: 150-161. [Crossref]

13. Nichols J, Zevnik B, Anastassiadis K, Niwa H, Klewe-Nebenius D, Chambers I, Schöler H, Smith A (1998) Formation of pluripotent stem cells in the mammalian embryo depends on the POU transcription factor. Cell 95:379-391.[Crossref]

14. Díez Villanueva P, Sanz-Ruiz R, Núñez García A, Fernández Santos ME, Sánchez PL, et al. (2012) Functional multipotency of stem cells: what do we need from them in the heart? Stem Cells Int 2012: 817364. [Crossref] 
15. Williams JM, Petersen BE (2008) Origin, evolution, and direction of human somatic cell therapy. In: Olmo DG, García-Verdugo JM, Alemany J, Gutiérrez-Fuente JA,(Eds.,) Cell Therapy. McGraw Hill-Interamericana. pp 3-14.

16. Serafini M, and CM Verfaille CM (2008) Multipotent progenitor cell populations obtained from bone marrow. In: Olmo DG, García-Verdugo JM, Alemany J, GutiérrezFuente JA, (Ed,.) Cell Therapy. McGraw Hill-Interamericana; pp. 89-102.

17. Boyer LA, Lee TI, Cole MF, Johnstone SE, Levine SS, et al. (2005) Core transcriptional regulatory circuitry in human embryonic stem cells. Cell 122: 947-956. [Crossref]

18. Hombría JC, Lovegrove B (2003) Beyond homeosis--HOX function in morphogenesis and organogenesis. Differentiation 71: 461-476. [Crossref]

19. Rieger CT, Fiegl M (2016) Microenvironmental oxygen partial pressure in acute myeloid leukemia: Is there really a role for hypoxia? Exp Hematol 44:578-582. [Crossref]

20. Ma T, Xie M, Laurent T, Ding S (2013) Progress in the reprogramming of somatic cells. Circ Res 112: 562-574. [Crossref]

21. Prasad A, Manivannan J, Loong DTB, Chua SM, Gharibani PM et al (2016) A review of induced pluripotent stem cell, direct conversion by transdifferentiation, direct reprogramming and oligodendrocyte differentiation. Regen Med 11: 181-191. [Crossref]

22. Gruh I, Martin U (2009) Transdifferentiation of stem cells: a critical view. Adv Biochem Eng Biotechnol 114: 73-106. [Crossref]

23. Eguizabal C, Montserrat N, Veiga A, Belmonte JCI (2013) Dedifferentiation, Transdifferentiation, and Reprogramming: Future Directions in Regenerative Medicine Semin Reprod Med 31: 082-094.[Crossref]

24. Hochedlinger K, Jaenisch R (2006) Nuclear reprogramming and pluripotency. Nature 441: 1061-1067. [Crossref]

25. Kim J, Efe JA, Zhu S, Talantova M, Yuan X, et al. (2011) Direct reprogramming of mouse fibroblasts to neural progenitors. Proc Natl Acad Sci U S A 108: 7838-7843. [Crossref]

26. Jopling C, Boue S, Izpisua Belmonte JC (2011) Dedifferentiation, transdifferentiation and reprogramming: three routes to regeneration. Nat Rev Mol Cell Biol 12: 79-89. [Crossref]

27. Iliakis G1, Wang Y, Guan J, Wang H (2003) DNA damage checkpoint control in cells exposed to ionizing radiation. Oncogene 22: 5834-5847. [Crossref]

28. Hartwell LH, Kastan MB (1994) Cell cycle control and cancer. Science 266: 18211828. [Crossref]

29. Nurse P (1997) Checkpoint pathways come of age. Cell 91: 865-867. [Crossref]

30. Paulovich AG, Toczyski DP, Hartwell LH (1997) When checkpoints fail. Cell 88: 315 321.[Crossref]

31. Rackham OJL, Firas J, Fang H, Oates ME, Melissa L, et al. (2016) A predictive computational framework for direct reprogramming between human cell types. 48 : 331-335. [Crossref]

32. Blomen VA, Boonstra J (2007) Cell fate determination during G1 phase progression. Cell Mol Life Sci 64: 3084-3104. [Crossref]

33. Dalton S (2015) Linking the Cell Cycle to Cell Fate Decisions. Trends Cell Biol 25: 592-600. [Crossref]

34. Roccio M, Schmitter1 D, Knobloch M, Okawa1 Y, Daniel Sage D, et al. (2013) Predicting stem cell fate changes by differential cell cycle progression patterns. Development 140: 459-470.[Crossref]

35. Fluckiger AC, Marcy G, Marchand M, Négre D, Cosset FL, et al. (2006) Cell cycle features of primate embryonic stem cells. Stem Cells 24: 547-556. [Crossref]

36. Filipczyk AA, Laslett AL, Mummery C, Pera MF (2007) Differentiation is coupled to changes in the cell cycle regulatory apparatus of human embryonic stem cells. Stem Cell Res 1: 45-60. [Crossref]

37. Cai S, Lee CC, Kohwi-Shigematsu T (2006) SATB1 packages densely looped, transcriptionally active chromatin for coordinated expression of cytokine genes. Nature Genetics 38: 1278-1288. [Crossref]

38. Reik W (2007) Stability and flexibility of epigenetic gene regulation in mammalian development. Nature 447: 425-432. [Crossref]
39. Matus DQ, Lohmer LL, Kelley LC, Schindler AJ, Kohrman AO et al (2011) Invasive Cell Fate Requires G1 Cell-Cycle Arrest and Histone Deacetylase-Mediated Changes in Gene Expression. Developmental Cell 35: 162-174. [Crossref]

40. Oliveira FM, Neumann E, Gomes MA, Caliari MV (2015) Entamoeba dispar: Could it be pathogenic. Trop Parasitol 5: 9-14. [Crossref]

41. Valenzuela O, Morán P, Ramos F, Cardoza JI, García G, et al. (2009) Two different chitinase genotypes in a patient with an amebic liver abscess: a case report. Am J Trop Med Hyg 80: 51-54. [Crossref]

42. Navaneethan U, Giannella RA (2011) Infectious colitis. Curr Opin Gastroenterol 27: 66-71. [Crossref]

43. Ali IK, Mondal U, Roy S, Haque R, Petri WA Jr, et al. (2007) Evidence for a link between parasite genotype and outcome of infection with Entamoeba histolytica. J Clin Microbiol 45: 285-289. [Crossref]

44. Ali IKM, Solaymani-Mohammadi S, Akhter J, Roy S, Gorrini C, Calderaro A, et al (2008) Tissue Invasion by Entamoeba histolytica: Evidence of Genetic Selection and/or DNA Reorganization Events in Organ Tropism. PLoS Negl Trop Dis 2: e219. [Crossref]

45. Kohler S, Tannich E (1993) A family of transcripts (K2) of Entamoeba histolytica contains polymorphic repetitive regions with highly conserved elements. Mol Biochem Parasitol 59: 49-58.[Crossref]

46. Haghighi A, Kobayashi S, Takeuchi T, Masuda G, Nozaki T (2002) Remarkable genetic polymorphism among Entamoeba histolytica isolates from a limited geographic area. $J$ Clin Microbiol 40: 4081-4090.[Crossref]

47. Ralston KS, Petri WA Jr (2011) Tissue destruction and invasion by Entamoeba histolytica. Trends Parasitol 27: 254-263.[Crossref]

48. Ghosh S, Frisardi M, Ramirez-Avila L, Descoteaux S, Sturm-Ramirez K, et al. (2000) Molecular epidemiology of Entamoeba spp.: evidence of a bottleneck (demographic Sweep) and transcontinental spread of diploid parasites. J Clin Microbiol 38: 38153821.[Crossref]

49. Bhattacharya A, Anand MT, Paul J, Yadav N, Bhattacharya S (1998) Molecular changes in Entamoeba histolytica in response to bacteria. J Eukaryot Microbiol 45: 28S-33S. [Crossref]

50. Galvan-Moroyoqui JM, Domínguez-Robles MdC, Franco E, Meza I (2008) The interplay between Entamoeba and enteropathogenic bacteria modulates epithelial cell damage. PLoS Negl Trop Dis 2: e266.[Crossref]

51. Gathiram V, Jackson TF (1985) Frequency distribution of Entamoeba histolytica zymodemes in a rural South African population. Lancet 7: 19-72. [Crossref]

52. Gathiram V, Jackson TF (1987) A longitudinal study of asymptomatic carriers of pathogenic zymodemes of Entamoeba histolytica. S Afr Med J 72: 669-672.[Crossref]

53. Meza I, de la Garza M, Meraz MA, Gallegos B, de la Torre M et al (1986) Isoenzyme patterns of Entamoeba histolytica isolates from asymptomatic carriers: use of gradient acrylamide gels. Am J Trop Med Hyg 35:1134-1139. [Crossref]

54. Irusen EM, Jackson TFHG, Simjee AE (1992) Asymptomatic intestinal colonization by pathogenic Entamoeba histolytica in amebic liver abscess: prevalence, response to therapy, and pathogenic potential. Clin Infect Dis 14: 889-893. [Crossref]

55. Biller L, Davis PH, Tillack M, Matthiesen J, Lotter H, et al. (2010) Differences in the transcriptome signatures of two genetically related Entamoeba histolytica cell lines derived from the same isolate with different pathogenic properties. BMC Genomics 11: 63-76. [Crossref]

56. Meyer M, Fehling H, Matthiesen J, Lorenzen S, Schuldt K, Bernin H, et al. (2016) Overexpression of differentially expressed genes identified in nonpathogenic and pathogenic Entamoeba histolytica clones allow identification of new pathogenicity factors involved in amoebic liver abscess formation. PLoS Pathog 12: e1005853. [Crossref]

57. Hackett AJ, Smith HS, Springer EL, Owens RB, Nelson-Rees WA (1977) Two syngeneic cell lines from human breast tissue: the aneuploid mammary epithelial (Hs578T) and the diploid myoepithelial (Hs578Bst) cell lines. JNCI J Natl Cancer Inst 58: 1795-1806. [Crossref]

58. Borowsky AD, Namba R, Young LJ, Hunter KW, Hodgson JG, et al. (2005) Syngeneic mouse mammary carcinoma cell lines: two closely related cell lines with divergent metastatic behaviour. Clin Exp Metastasis 22: 47-59. [Crossref] 


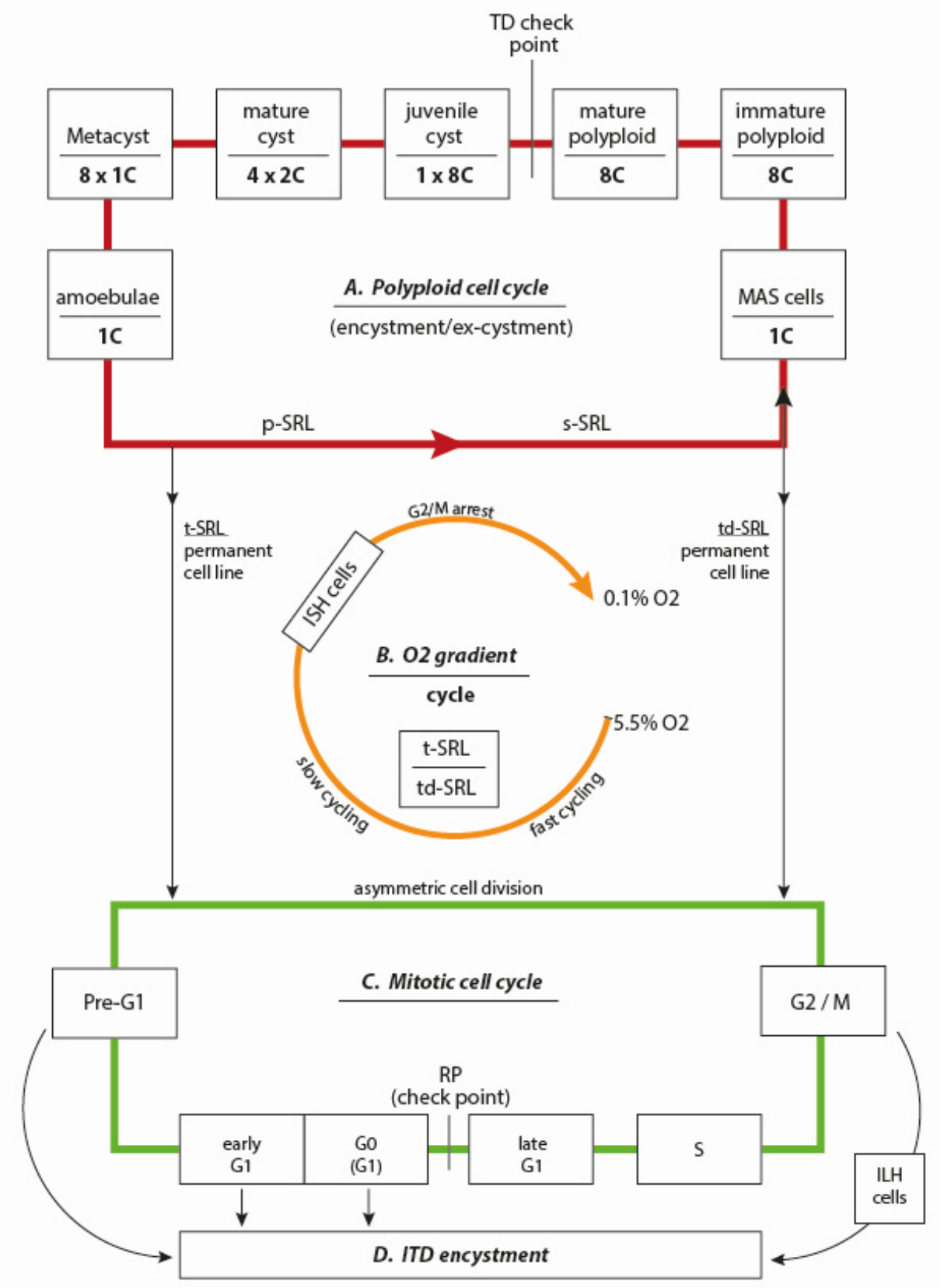

Figure S1. The intestinal life cycle of E. invadens and the differentiation potential of self-renewing respectively quiescent cells as observed in OCB cultures.

A. The polyploid cell cycle (polyploidisation-depolyploidisation cycle): MAS precursor cells committed for ATD encystment are produced by the s-SRL line; they endoreplicate forming eight genome copies. To transit the TD checkpoint and form cysts polyploid MAS precursor cells need maturation. During ex-cystment the octoploid innercyst cell hatches out and form the metacyst. The metacyst segregates its eight genome replicas in eight amoebulae. Amoebulae generate the primary stem cell line p-SRL which converses into the secondary more oxygenic s-SRL. Both short leaving cell lines proliferate by asymmetric cell division. The s-SRL cell line produces new MAS cells that restart the polyploid cell cycle; $\boldsymbol{B}$. The oxygen gradient cycle: the ubiquitous "immortal" cell lines t-SRL and td-SRL proliferate both in the upper and lower oxygen zones of the intestinal oxygen gradient. In strong hypoxic conditions the t-SRL/tdSRL lines switch from asymmetric to symmetric cell fate forming identical ISH cells. ISH cells transit the G1 checkpoint and arrest at the G2 check point. They return to asymmetric cell fate when hypoxia decreases; $\boldsymbol{C}$. Mitotic cell cycle and ITD encystment: Mitoticly uncommitted cells prior restriction point RP and quiescent G0 cells may be directly induced to terminal differentiation (ITD encystment). Committed cells transiting the RP check point do not form ITD cysts. G2 and M cells divide in hypoosmotic non-nutrient media to identical daughter cells (ILH cells) that form ITD cysts.

Copyright: $@ 2017$ Niculescu VF. This is an open-access article distributed under the terms of the Creative Commons Attribution License, which permits unrestricted use, distribution, and reproduction in any medium, provided the original author and source are credited. 